\title{
Mangiferin Induces Apoptosis by Regulating Bcl-2 and Bax Expression in the CNE2 Nasopharyngeal Carcinoma Cell Line
}

\author{
Li-Li Pan, Ai-Yan Wang, Yong-Qi Huang, Yu Luo, Min Ling*
}

\begin{abstract}
To investigate the anti-proliferative mechanism of mangiferin in a human nasopharyngeal carcinoma cell line, CNE2 cells were incubated with different concentrations of mangiferin $(12.5,25,50,100,150$ and $200 \mu \mathrm{M})$ or with PBS as a control for $\mathbf{7 2}$ hours. Analyses were made of the cell cycle and apoptosis with measurement of mRNA and protein levels of two apoptosis-related genes, Bcl-2 and Bax. Flow cytometry assays showed mangiferin could inhibit CNE2 cell proliferation via G2/M arrest and induction of early apoptosis. Real time PCR and Western blotting showed the mRNA and protein level of Bcl-2 to be down-regulated, while those of Bax were up-regulated, when CNE2 cells were treated with mangiferin. This investigation indicated anti-proliferation effects of mangiferin through induction of cell apoptosis regulated by Bcl-2 and Bax expression.
\end{abstract}

Keywords: Mangiferin - CNE2 - apoptosis - Bcl-2 - Bax

Asian Pac J Cancer Prev, 15 (17), 7065-7068

\section{Introduction}

Mangiferin, a C-glucosyl xanthone (1, 3, 6, 7 -tetrahydroxyxanthone -C2- $\beta$-D -glucoside), is a natural flavonoid compound widely distributed in a variety of plant families, such as Mangifera indica, Mangifera odorata, Gentiana lutea, Pyrrosia gralla and Rhizoma anemarrhenae. The easiest source of mangiferin is mango plant (Mangifera indica), especially the leaves. Mangiferin is also detected at moderate concentration in bark, fruits, and roots, and at the most highly concentration in young mango leaves (Barreto et al., 2008). The mango tree is indigenous to the Indian. It was introduced into China in the Tang Dynasty, has been cultivated in South China for over 1000 years (Wang et al., 1997).

The chemistry of this nature compound, mangiferin, has been relatively well understood. Previous studies demonstrated that it exhibit numerous biological activities, including anti-inflammatory, anti-proliferative, antioxidative, anti-diabetic and neuroprotective effects (Ichiki et al., 1998; Yoshimi et al., 2001; Muruganandan et al., 2002).

Some studies in leukemia cells have showed that the anti-cancer effect of mangiferin was regulated through apoptosis. For example, in HL-60 human acute myeloid leukemia cells, mangiferin induced apoptosis by suppressing expression of Bcl-xL and XIAP and nuclear entry of NF- $x$ B (Kaori et al., 2011). In K562 human leukemia cells, mangiferin has been found to inhibit telomerase activity and induce apoptosis (Cheng et al., 2007). Recently, anti-proliferative effect of mangiferin was also observed in CNE2 human nasopharyngeal carcinoma cells, which leads to efficient induction of apoptosis (Yang et al., 2009). However, the molecular mechanisms of the anti-cancer effect have not been fully elucidated. In this study, we aim to investigate the inhibitory effects of mangiferin in CNE2 cells and to explore the possible underlying mechanisms.

\section{Materials and Methods}

\section{Materials}

Mangiferin was provided by Guangxi Minzu Hospital, China. The concentration was measured by CZEP capillary zone electrophoresis as described previously (Nong et al., 2005). Mangiferin was dissolved in dimethyl sulfoxide (Sigma, USA) and filtered through $0.45 \mu \mathrm{m}$ syringe filters (Millipore, USA). Then stock solution at $200 \mu \mathrm{M}$ was prepared for analysis. Human nasopharyngeal carcinoma cell line CNE2 was obtained from Guangxi Medical University, China. Cells were cultured in RPMI1640 medium (Gibco, USA) supplemented with $10 \%$ fetal calf serum (Gibco, USA), $100 \mu \mathrm{g} / \mathrm{ml}$ penicillin (Gibco, USA), and $100 \mathrm{U} / \mathrm{ml}$ streptomycin (Gibco, USA) at $37^{\circ} \mathrm{C}$ in an atmosphere containing $5 \% \mathrm{CO}_{2}$. When reaching 60\%-80\% confluence, cells were trypsinized with $0.25 \%$ trypsin (Amresco, USA). Good condition cells were selected for further experiments.

Flow cytometry assay of apoptosis and cell cycle

To estimate early apoptotic cell number, CNE2 cells were plated in 6-well plates at 100000 cells/well and 
incubated with various concentrations of mangiferin (12.5, $25,50,100,150$ and $200 \mu \mathrm{M}$ ) or with PBS as control. After incubation for 72 hours, cells were collected and washed with ice-cold PBS and binding buffer, and incubated with AnnexinV-PE (Beckman Coulter, USA) on ice in dark for at least 15 minutes. Propidium iodide (PI) (Beckman Coulter, USA) was added on ice in dark for at least 15 minutes. All samples were then analyzed on a FACS Calibur flow cytometer (Beckman Coulter Inc. USA) to determine the cell apoptosis rate and the cell number at each cell cycle.

\section{$R N A$ extraction and real-time fluorescent quantitative $R T$-PCR}

$\mathrm{CNE} 2$ cells were treated with various concentrations of mangiferin or with PBS as control for 72 hours, collected by centrifugation, and washed twice with icecold PBS. Total RNA was isolated using RNAiso Plus reagent (TaKaRa, China) according to the manufacturer's recommendations. cDNA was synthesized from $2 \mu \mathrm{g}$ of total RNA. All reverse transcription was performed with random hexanucleotide using the PrimeScript RT reagent kit with gDNA Eraser (TaKaRa, China). Table 1 shows the primers used in this study. After adding fluorescent probes, real-time PCR was done using a Mastercycler ep realplex real time PCR meter (Eppendorf, Germany) with 40 cycles of denaturation at $95^{\circ} \mathrm{C}$ for 30 seconds, annealing at $58^{\circ} \mathrm{C}$ for 30 seconds, and extension at $68^{\circ} \mathrm{C}$ for 30 seconds. Relative transcription levels were calculated with the following formula: Relative quantitation $=2^{-\triangle \Delta \mathrm{Ct}}$ ( $\triangle \triangle \mathrm{Ct}=(\mathrm{Ct}$ mangiferin-treated group- $\mathrm{Ct}$ control group) Destination gene - (Ct mangiferin-treated group-Ct control group) GAPDH).

\section{Western blot analysis}

$\mathrm{CNE} 2$ cells were treated with various concentrations of mangiferin or with PBS as control for 72 hours, collected by centrifugation, washed twice with ice-cold PBS, and resuspended in $200 \mu 1$ protein lysate. Cells lysates were centrifuged at $12000 \mathrm{r} /$ minute for 15 minutes at $4^{\circ} \mathrm{C}$ and the supernatant was stored at $-20^{\circ} \mathrm{C}$. The protein concentration was determined by BCA Protein Assay Kit (Beyotime Biotech, China). $40 \mu \mathrm{g}$ protein was electrophoresed on a $12 \%$ polyacrylamide gel containing SDS (SDS-PAGE) and transferred onto nitrocellulose membranes (Millipore, USA) at $4^{\circ} \mathrm{C}$ for 4 hours. Membranes were blocked in $5 \%$ non-fat milk for 1 hour followed by incubation with primary antibodies of Bax and Bcl-2 (1:1000) Santa Cruz Biotechnology, USA) overnight at $4^{\circ} \mathrm{C}$ with shaking. House-keeping gene $\beta$-actin was used as an internal standard. After washing in Tris-buffered saline with Tween

Table 1. Gene-specific Primers for RT-PCR

\begin{tabular}{lll}
\hline Name & Orientation & Sequence of primer \\
\hline Bcl-2 & Sense & 5'-TTGGATCAGGGAGTTGGAAG-3' \\
& Anti-sense & 5'- TGTCCCTACCAACCAGAAGG-3' \\
\multirow{2}{*}{ Bax } & Sense & 5'- CGTCCACCAAGAAGCTGAGCG-3' \\
& Anti-sense & 5'- CGTCCACCAAGAAGCTGAGCG-3' \\
& & 5'-AGCACTCCCGCCACAAAGATG-3' \\
GAPDH & Sense & 5'-ACGGATTTGGTCGTATTGGG-3' \\
& Anti-sense & 5'- TGATTTTGGAGGGATCTCGC-3' \\
\hline
\end{tabular}

GAPDH: glyceraldehyde-3-phosphate dehydrogenase
20 (TBST), membranes were incubated with peroxidaselinked IgG conjugates for 2 hours, washed with TBST, and bands were visualized by an enhanced chemiluminescence reagent kit (Beyotime Biotech, China). The relative band intensity was determined by a gel image analysis system (Bio-Rad, USA) and normalized with $\beta$-actin.

\section{Statistical analysis}

Statistical analyses were done using the SPSS 13.0 software package. All experiments were repeated at least three times. Results of multiple experiments are expressed as mean \pm standard deviation (SD). The statistical analysis involving two groups was made by Student's t test. A $p$ value less 0.05 was considered significant.

\section{Results}

\section{Cell apoptosis induced by mangiferin}

Cell apoptosis and cell cycle phases was analysed by flow cytometry using annexin V-PE/PI staining. The percentage of early apoptotic cells and percentage of cells on different cell cycle were listed in Table 2 . We found that mangiferin could induce CNE2 cells apoptosis in a dose-

Table 2. Apoptosis and Cell Cycle Phases of CNE2 Treated with Mangiferin

\begin{tabular}{ccccc}
\hline $\begin{array}{c}\text { Mangiferin } \\
(\mu \mathrm{M})\end{array}$ & $\begin{array}{c}\text { Apoptosis } \\
(\%)\end{array}$ & \multicolumn{3}{c}{ Phase in cell cycle $(\%)$} \\
\hline 0 & $4.6 \pm 1.6$ & $26.8 \pm 3.5$ & $8.5 \pm 1.4$ & $2.2 \pm 0.3$ \\
12.5 & $20.5 \pm 2.1^{* *}$ & $73.0 \pm 0.8^{*}$ & $9.1 \pm 1.0^{*}$ & $10.5 \pm 0.8^{* *}$ \\
25 & $39.6 \pm 1.8^{*}$ & $72.9 \pm 0.6^{*}$ & $9.2 \pm 0.5^{*}$ & $11.5 \pm 0.9^{*}$ \\
50 & $40.6 \pm 1.3^{*}$ & $70.5 \pm 0.7^{*}$ & $10.1 \pm 1.3^{*}$ & $12.4 \pm 0.9^{*}$ \\
100 & $41.3 \pm 2.4^{*}$ & $69.1 \pm 1.1^{*}$ & $10.7 \pm 1.2^{*}$ & $13.0 \pm 1.0^{*}$ \\
150 & $42.7 \pm 4.6^{*}$ & $70.0 \pm 1.0^{*}$ & $11.7 \pm 1.0^{*}$ & $13.6 \pm 1.1^{*}$ \\
200 & $44.1 \pm 2.6^{* *}$ & $67.8 \pm 1.0^{*}$ & $12.3 \pm 0.9^{*}$ & $14.8 \pm 1.2^{* *}$ \\
\hline
\end{tabular}

Values are mean $\pm \mathrm{SD}$. ${ }^{*} \mathrm{p}<0.05$ vs Control group; ${ }^{*} \mathrm{p}<0.05$ vs Other different groups

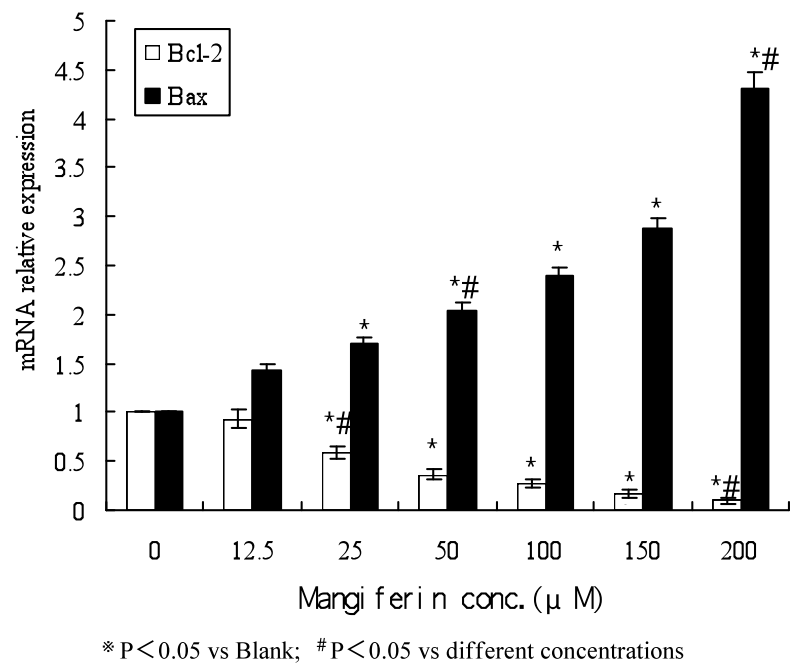

Figure 1. mRNA Level of Bcl-2 and Bax Analyzed by Real Time PCR. CNE2 Cells were Treated with Various Concentrations of Mangiferin for $\mathbf{7 2}$ hours. Total RNA was isolated for real-time PCR analysis. Values are mean \pm SD. ${ }^{*} p<0.05$ vs Control group; ${ }^{*} p<0.05$ vs Other different groups. Compared with control cells, the mRNA level of Bcl-2 decreased, and the mRNA level of Bax increased with the treatment of mangiferin in a dose-dependent manner 


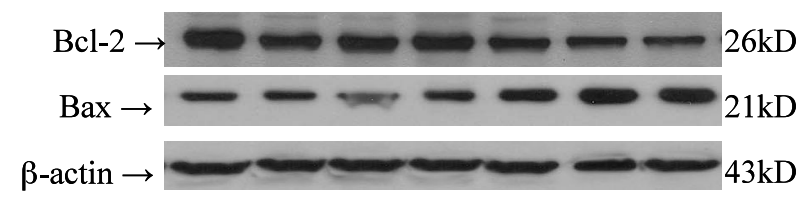

Mangiferin : $0 \quad 12.5 \quad 25 \quad 50 \quad 100 \quad 150 \quad 200 \mu \mathrm{M}$

Figure 2. Protein Level of Bcl-2 and Bax Analyzed by Western Blot. CNE2 cells were treated with various concentrations of mangiferin for $72 \mathrm{hr}$. Protein was separated on a $12 \%$ SDS-PAGE and transferred to membrane for western blot. Compared with control cells, the protein level of Bcl-2 decreased and the level of Bax increased with the treatment of mangiferin in a dosedependent manner

dependent manner. In addition, mangiferin could inhibit CNE2 cells proliferation via $\mathrm{G} 2 / \mathrm{M}$ arrest and induction of early cell apoptosis.

\section{The effect of mangiferin on Bcl-2 and Bax}

We further analyzed the mRNA and protein level of Bcl-2 and Bax in cells treated with various concentrations of mangiferin. We found down-regulation of Bcl-2 mRNA level and up-regulation of Bax mRNA level in mangiferin-treated cells compared with control (Figure 1). Consistently, the protein level of Bcl-2 was downregulated and the protein level of Bax was up-regulated in mangiferin-treated cells compared with control (Figure 2).

\section{Discussion}

Nasopharyngeal carcinoma (NPC), a distinct type of head and neck cancer, has its highest incidence rates in southern China and southeastern Asia (Jemal et al., 2011). In conventional clinical NPC therapy, radiotherapy is the most commonly used, followed by combined chemotherapy (Lee et al., 2012). Unfortunately, the incidence of cancer cell resistance to chemotherapy drug is quite common, which limits the use of chemotherapy as a treatment option. Therefore, it is important to find new therapeutic targets.

Recently, ZD1839 (gefitinib) or metformin has been shown to enhance the effectiveness of chemotherapy for NPC when it combined with chemotherapy drugs, cisplatin (Gu et al., 2013; Sun et al., 2014). However, these synthetic drugs might cause some adverse effects in vivo (Ieki et al., 2003; Waleed, 2007), so natural compounds are considered to be better therapeutic reagents for NPC at present. One of the promising natural compounds with anti-cancer activity and chemosensitizing activity is mangiferin (Louisa et al., 2014).

Previous studies have indicated that mangiferin may inhibit cancer cell growth through inducing cell apoptosis in several cell studies. For example, mangiferin has been found to block human leukemia K562 cells mitosis, inhibit cell proliferation, and induce cells apoptosis (Cheng et al., 2007). Similarly, mangiferin could inhibit BEL-7404 human hepatocellular carcinoma cells proliferation via G2/M arrest and induce the cells apoptosis (Huang et al., 2002). Therefore, our finding of cell growth inhibition via $\mathrm{G} 2 / \mathrm{M}$ arrest and induction of CNE2 cells apoptosis is consistent with previous reports, further suggesting that apoptosis induction might be one underlying anti-cancer mechanism of mangiferin.

Apoptosis is a complex process regulated by a variety of factors, such as Bcl-2 and Bax. Currently, there are two widely accepted classical signaling pathways of cell apoptosis, including the mitochondrial pathway and the cell death receptor pathway (Jegham et al., 2010). Proteins of the Bcl-2 family have been shown to regulate the mitochondrial pathway by controlling the permeabilization of the outer mitochondrial membrane. The Bcl-2 protein, a potent inhibitor of apoptotic cell death that locates in the mitochondrial membrane, endoplasmic reticulum and outer nuclear membrane, has been shown to decrease cell shrinkage, chromatin condensation, and DNA cleavage through preventing early stage of cell apoptosis program (Haidara et al., 2006). In response to many types of stress or damage, certain members of the Bcl-2 family are activated to cause the activation of $\mathrm{Bax}$ at the mitochondrion. The $\mathrm{Bax} / \mathrm{Bcl}-2$ ratio has been suggested to be one key factor to determine whether a cell could enter the apoptotic program (Karmakar et al., 2007). In the present study, we found down-regulated Bcl2 mRNA and protein levels and up-regulated Bax mRNA and protein levels in mangiferin-treated cells compared with control, suggesting that $\mathrm{Bcl}-2$ and $\mathrm{Bax}$ are involved in the regulation of early cell apoptosis.

Our experimental evidences clearly indicate that mangiferin could inhibit CNE2 cells proliferation via $\mathrm{G} 2 / \mathrm{M}$ arrest and induce cell apoptosis by regulating Bcl-2 and Bax expression. More studies in other cancer cells are needed to confirm our conclusion and to better understand the underlying anti-cancer mechanisms of mangiferin.

\section{Acknowledgements}

This work was supported by grant from the Research Project of Guangxi Colleges and Universities (201106LX097).

\section{References}

Barreto JC, Trevisan MT, Hull WE et al (2008). Characterization and quantitation of polyphenolic compounds in bark, kernel, leaves, and peel of mango (Mangifera indica). J Agric Food Chem, 56, 5599-610.

Cheng P, Peng ZG, Yang J et al (2007). The effect of mangiferin on telomerase activity and apoptosis in leukemic K562 cells. Zhong Yao Cai, 30, 306-9.

Gu WG, Huang Y, Yuan ZY et al (2013). ZD1839 and cisplatin alone or in combination for treatment of a nasopharyngeal carcinoma cell line and xenografts. Asian Pac J Cancer Prev, 14, 1787-90.

Huang HY, Nong CZ, Guo LX et al (2002). The proliferation inhibition effect and apoptosis induction of Mangiferin on BEL-7404 human hepatocellular carcinoma cell. Zhong Hua Xiao Hua Za Zhi, 22, 341-3.

Haidara K, Zamir L, Shi QW et al (2006). The flavonoid Casticin has multiple mechanisms of tumor cytotoxicity action. Cancer Lett, 242, 180-90.

Ichiki H, Miura T, Kubo M et al (1998). New antidiabetic compounds, mangiferin and its glucoside. Biol Pharm Bull, 21, 1389-90. 
Ieki R, Saitoh E, Shibuya M (2003). Acute lung injury as a possible adverse drug reaction related to gefitinib. Eur Respir J, 22, 179-81.

Jegham H, Roy J, Maltais R et al (2010). A novel aminosteroid of the $5 \alpha$-androstane- $3 \alpha, 17 \beta$-diol family induces cell cycle arrest and apoptosis in human promyelocytic leukemia HL60 cells. Invest New Drugs, 30, 176-85.

Jemal A, Bray F, Center MM, et al (2011). Global cancer statistics. CA Cancer J Clin, 61, 69-90.

Karmakar S, Banik NL, Ray SK et al (2007). Curcumin suppressed anti-apoptotic signals and activated cysteine proteases for apoptosis in human malignant glioblastoma U87MG cells. Neurochem Res, 32, 2103-13.

Kaori Shoji, Masanobu Tsubaki, Yuzuru Yamazoe et al (2011). Mangiferin induces apoptosis by suppressing Bcl-xL and XIAP expressions and nuclear entry of NF- $x \mathrm{~B}$ in HL-60 Cells. Arch Pharm Res, 34, 469-75.

Lee AW, Lin JC, Ng WT (2012). Current management of nasopharyngeal cancer. Semin Radiat Oncol, 22, 233-44.

Louisa M, Soediro TM, Suyatna FD (2014). In vitro modulation of P-glycoprotein, MRP-1 and BCRP expression by mangiferin in doxorubicin-treated MCF-7 cells. Asian Pac J Cancer Prev, 15, 1639-42.

Muruganandan S, Gupta S, Kataria M et al (2002). Mangiferin protects the streptozotocin-induced oxidative damage to cardiac and renal tissues in rats. Toxicology, 176, 165-73.

Nong CZ, He WS, Debbi F et al (2005). Capillary electrophoresis analysis of mangiferin extracted from Mangifera indica. Journal of Chromatography B, 826, 226-31.

Sun XJ, Zhang P, Li HH et al (2014). Cisplatin combined with metformin inhibits migration and invasion of human nasopharyngeal carcinoma cells by regulating E-cadherin and MMP-9. Asian Pac J Cancer Prev, 15, 4019-23.

Wang JL, Guan ZX, Zhang HZ (1997). Studies on current status of mango resources and the processing technology in China. Natural Resources, 11, 52-9.

Sweileh WM (2007). Contraindications to metformin therapy among patients with type 2 diabetes mellitus. Pharm World Sci, 29, 587-92.

Yang XL , Liu XC, Lan J et al (2009). Effects of Mangiferin on proliferation, apoptosis and cycle of nasopharyngeal carcinoma CNE2 cells. Shangdong Medical, 49, 23-4.

Yoshimi N, Matsunaga K, Katayama M et al (2001). The inhibitory effects of mangiferin, a naturally occurring glucosylxanthone, in bowel carcinogenesis of male F344 rats. Cancer Lett, 163, 163-70. 